\title{
Pre-Processing and Image Enhancement Techniques
}

\author{
A. Sivaramakrishnan ${ }^{1}$, M. Vinoth Kumar ${ }^{2}$ \\ Assistant Professor, Department of Computer Science, DMI St.Eugene University, Lusaka, Zambia ${ }^{1}$ \\ Lecturer, Department of Computer Science, DMI St.Eugene University, Lusaka, Zambia ${ }^{2}$
}

\begin{abstract}
This article deals with pre-processing and enhancement activities such as removal of film artifacts and labels, filtering the image. A gradient based image enhancement method for mammography and MRI images is based on the first derivative and the local statistics. The proposed mammogram image enhancement method, the film artifacts such as labels and X-ray marks are removed and the high frequency components are removed using various filtering. The performance of the proposed method is also evaluated by means of Signal-to Noise-Ratio (SNR) using the mammographic images from the Mammography Image Analysis Society database and MRI brain images obtained from KOVAI Medical Center Hospital (KMCH), India.
\end{abstract}

Keywords: MIAS, MRI, Artifacts, Filter, PSNR, ASNR.

\section{INTRODUCTION}

Obtaining real breast mammogram images for carrying out research is highly difficult due to privacy issues, legal issues and technical hurdles. Hence, the MIAS database is used to study the efficiency of the proposed intelligent system since it is a benchmark database available online for research.

\section{MIAS DATABASE}

The MIAS, which is an organization of UK-based research groups interested in the understanding of mammograms, has produced a digital mammography database (ftp://peipa.essex.ac.uk). The X-ray films in the database have been carefully selected from the United Kingdom National Breast Screening Programme and digitized with a Joyce-Lobel scanning microdensitometer to a resolution of $50 \mu \mathrm{m}$ x $50 \mu \mathrm{m}, 8$ bits representing each pixel. Table 1.1 and Figure 1.1 summarize the different categories of mammograms in MIAS database.

\section{PAGE STYLE}

Table 1.1 MIAS Category

\begin{tabular}{|l|l|l|l|}
\hline Sl.No & MIAS Category & Image Labels & No. of Image Pairs \\
\hline 1 & Normal & $\begin{array}{l}002,004,017-028,031,033-034,037,039,043-044, \\
047,057,068-070,077,081,087,115,129-31,138-144, \\
146-155,159-161\end{array}$ & 53 \\
& & $\begin{array}{l}001,003,005-006,008-014,030,035,040,046,066, \\
071,122,135,145,158\end{array}$ & 21 \\
\hline 2 & $\begin{array}{l}\text { Circumscribed } \\
\text { masses }\end{array}$ & $105-114,116-121,123-128$ & 22 \\
\hline 3 & Calcifications & $145-148,175-188,189-208$ & 19 \\
\hline 4 & Spiculated masses & $58-65,75-76,78-80,82-86$ & 18 \\
\hline 5 & Architectural & $036,038,041-042,045,048-056$ & 14 \\
\hline 6 & Asymmetries & $007,015-016,029,032,067,072,132-134,136-137,156-$ & 14 \\
& & 167 & \\
\hline 7 & Miscellaneous & \multicolumn{1}{|c|}{ TOTAL } & 161 \\
& \multicolumn{2}{|l}{}
\end{tabular}




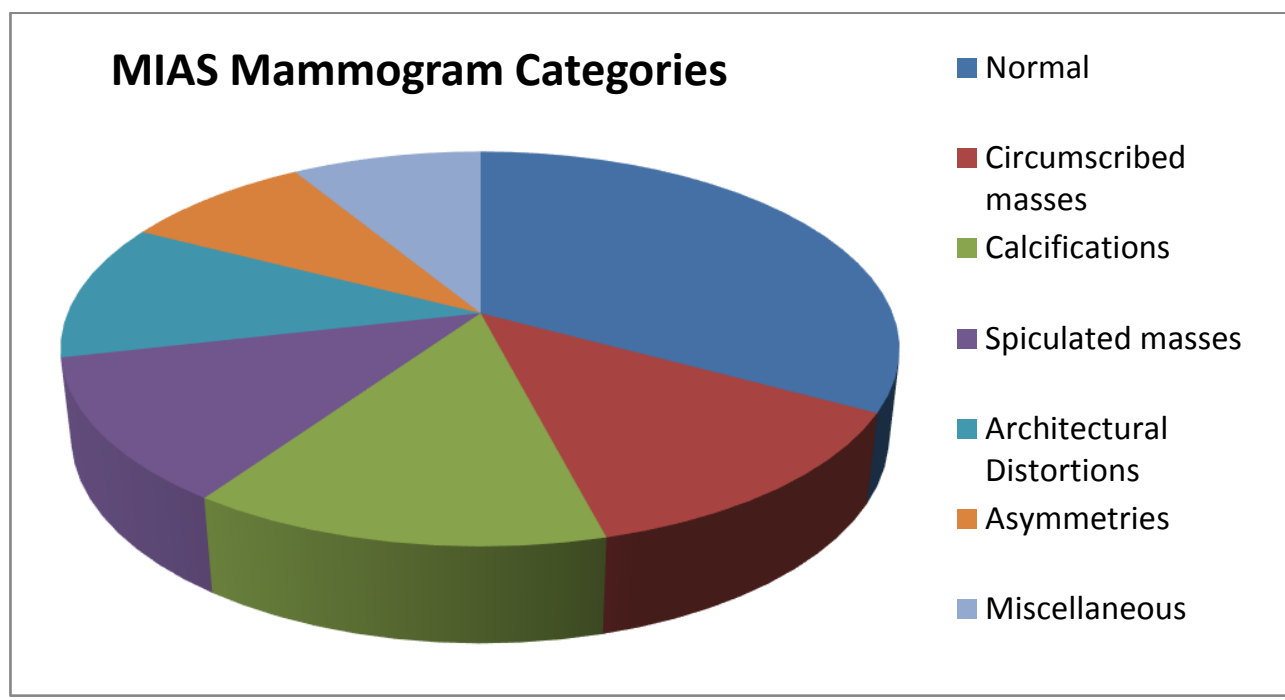

Figure 1.1 MIAS mammogram categories of 161 pairs

The MIAS Digital Mammogram Database contains 322 images Mediolateral Oblique representing 161 bilateral mammogram pairs. The database is divided into seven categories such as massess, circumscribed masses, spiculated lesions, ill-defined masses, architectural distortion and asymmetric densities of normal and abnormal mammograms. Figure 1. 2 shows the Sample Mammogram from MIAS Database

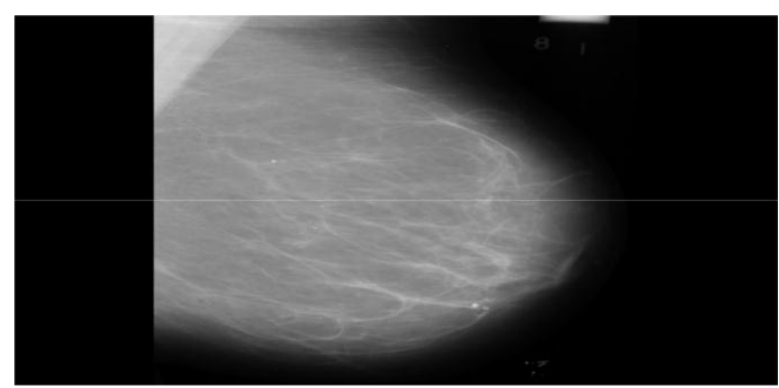

Figure 1.2 Mammogram from MIAS Database

\section{MRI IMAGE}

Preprocessing depends on the procedure and corrects system abnormalities such as differential light detection efficiency, dead pixels or dark noise. Processing is control of the raw data just after acquisition. A raw 3-D MRI image comes as a $3 \mathrm{D}$ array of pixels. Each pixel has a grey scale range from 0 to 65535 in the 16 -bit pixel case or 0 to 255 in the 8 -bit pixel case. Most medical imaging systems generate images using 16-bit grayscale range.

This proposed system for pre-processing and enhancement through Magnetic Resonance Image (MRI) is a gradient based image enhancement method and is based on the first derivative, local statistics. It is corporate with two stages of preparation. In the first stage, the MRI brain image is given to the preprocessing and enhancement stage, where the film artifacts and high frequency components are removed. In the second stage the performance of enhancement filters are measured and the best evaluated filter is selected for further enhancement.

\section{Brain Database}

In the medical world, to ingress the real medical images like MRI, PET or CT scan and to resume a research are very complicated because of privacy problem and heavy technical deadlocks. The objective of this study is to compare enhancement filters for detection of brain tumor through Brain MRI. Generally the proposed work can be implemented in a hospital by maintaining patients MRI in a database which can be available in Linux Network through LAN (Local Area Network) for easy access. for example, Kovai Medical Center Hospital (KMCH), India. They acquire all patients MRI acquired from 0.5T intra operative Magnetic Resonance scanner and distributed it immediately in their own LAN. The proposed work only focus on T2 weighted images and its size like $256 \times 256 \times 58(0.86 \mathrm{~mm}, 0.86 \mathrm{~mm}, 2.5 \mathrm{~mm})$. The following table shows the properties of MRI brain image. Table 1.2 shows the MR Brain Image- properties 
Vol. 9, Issue 7, July 2020

Table 1.2 MR Brain Image- properties

\begin{tabular}{|c|l|c|}
\hline S.No & Properties & Detail \\
\hline 1 & Thickness & $1 \mathrm{~mm}$ \\
\hline 2 & Resolution & $1 \times 1 \mathrm{~mm}$ \\
\hline 3 & TR(Repetition Time) & 400 \\
\hline 4 & TE(Echo Time) & $16 \mathrm{~ms}$ \\
\hline 5 & FOV (Field of view) & $220 * 220 \mathrm{~mm}$ \\
\hline
\end{tabular}

This hospital offers the possibility to acquire 4000 MR images in 3 minutes and 40 seconds and similar images acquired from 1.5T Conventional Scanner. But this scanner enclose the drawback of intra-operative image is that slice remains thick $(2.5 \mathrm{~mm})$. These database contains patients image data from the age 20 .

The following figure 1.3 shows the sample patient brain MRI's from database.

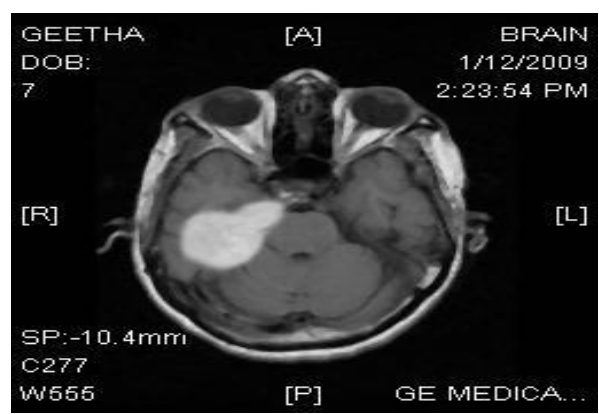

Fig. 1.3 MRI- KMCH Database

\section{REMOVAL OF FILM ARTIFACTS}

The film artifacts are removed using tracking algorithm. Starting from the first row and first column of the image, the intensity value of the pixel is copied into a new two-dimensional array when the following conditions are satisfied. Initially a flag value is assigned as zero. If the intensity value is zero then it is copied to the new image. If the intensity value is greater than zero, it is copied to a new image and the flag value is set as one. Again the next value is compared: if it is a non-zero value then that value is stored in a new array; if it is zero, the flag value is set as zero and the remaining pixels in that row are skipped. The next iteration is started with the next row. In this manner, only the brain and breast region is copied into the new array; thus the film artifacts are removed from the image. Figure 1.4 and figure 1.5 shows the removal of film artifacts
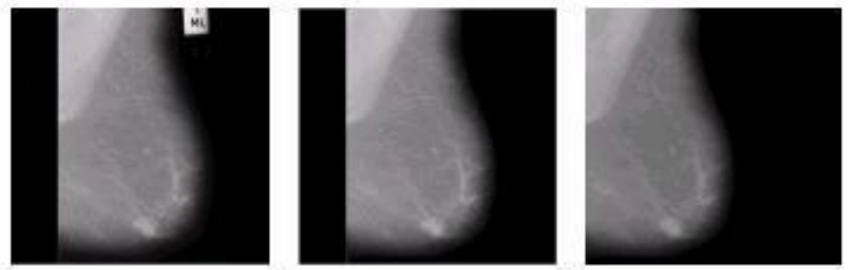

Figure 1.4 Original mammogram Removal of film artifacts
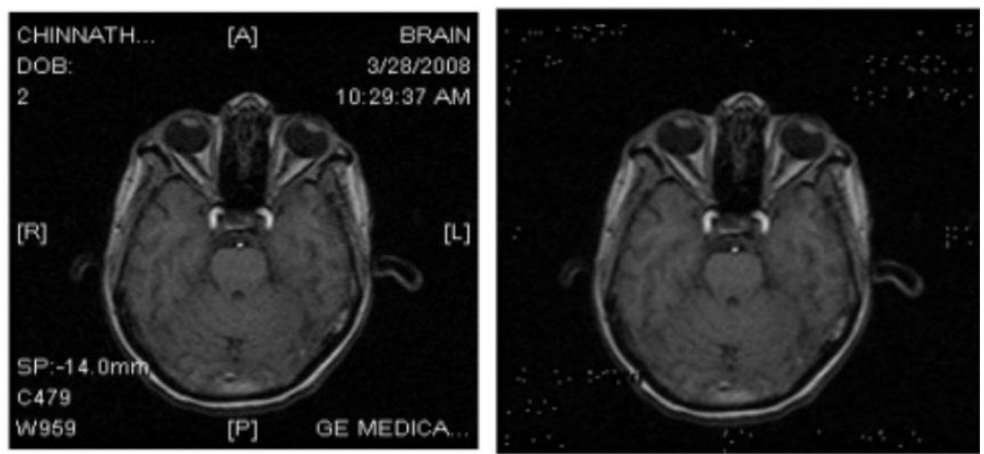

Figure 1.5 Original Image after Removal of Film-Artifacts 


\section{IMAGE ENHANCEMENT}

Image enhancement methods examine about how to enhance the visual presence of mammogram and MRI images from database and the contrast enhancing brain volumes were linearly regulate. The enhancement activities are removal of high frequency components and unwanted labels, for filtering the Images. Image enhancement is one of the main processing techniques.

It can handle as transforming one image to another and it to enhance the intelligible or perception of it for human observers or to extract neat details of images which may produce suitable input for other automated image analysis systems (Apurba et al. 2011). The proposed system describes the detail of Enhancement using three types of filters such as Median Filter, Weighted Median Filter and Adaptive and Spatial filter for removing high frequency components.

\section{A. Median Filter}

Median Filter can erase the unwanted stuff's on the MRI like noise, the high frequency components from MRI without disturbing the edges, band width of the image etc. and is used to reduce salt and pepper noise. This method calculates the median of the neighboring pixels to determine the new and de-noised value of the pixel. A median is computed by sorting all pixel values by their size, then choosing the median value as the new value for the pixel. Thangavel and karnan (2005)[38] said median filtering has been found to be very powerful in removing noise from two dimensional signals without blurring edges.

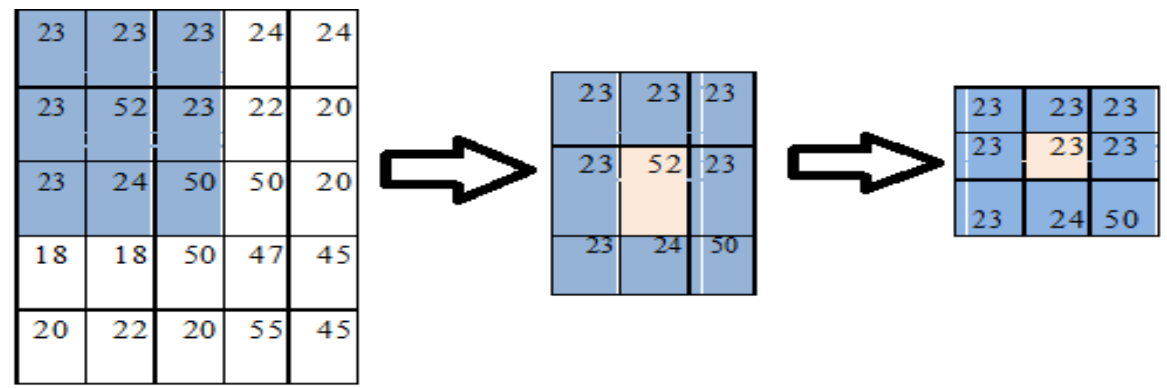

Figure 1.6 Median filtering

Let us consider first $3 \times 3$ windows there are nine pixels, the pixel intensity values are 23, 23, 23, 23, 52, 23, 23, 24, and 50. The values are arranged in ascending order such as 23, 23, 23, 23,23,23,24,50 and 52. The median is equal to 23 . The center pixel value is replaced with 23. This procedure is followed for all the pixels in the image to smoothen the entire MRI Image. For each pixel, 3x3, 5x5, 7x7, 9x9 and 11x11 Windows of Neighborhood Pixels are extracted and the median value is calculated for the window. The median value is placed with instead of intensity value of the center pixel. High Resolution Image is obtained when the process is repeated.

The tumor pixels in the foreground region are white in color their Intensity is 0 to 255 and the background region is normally black in color their intensity is 0 to 10 . The Contrast of the region is defined by

$\mathrm{C}=(\mathrm{f}-\mathrm{b}) /(\mathrm{f}+\mathrm{b})$

Where $\mathrm{f}$ is the mean gray-level value of the foreground $\mathrm{b}$ is the mean gray-level value of the background

\section{B. Weighted Median Filter}

The Weighted Median Filter is to remove high frequency component noise from MRI Image without disturbing the edges. The weighted median filter is a variation of the median filter that incorporates spatial information of the pixels when computing the median value. A weighted median filter is implemented as follows:

$\mathrm{W}(\mathrm{x}, \mathrm{y})=\operatorname{median}\{\mathrm{w} 1 \times \mathrm{x} 1 \ldots \mathrm{wn} \times \mathrm{wn}\}$

$\mathrm{x} 1 \ldots . . \mathrm{xn}$ are the intensity values inside a window centered at $(\mathrm{x}, \mathrm{y})$ and $\mathrm{w} \times \mathrm{n}$ denotes replication of $\mathrm{x}, \mathrm{w}$ times.

The Weighted Median Filter methodology is same as Median Filter but here, giving Weights to the pixels. If the intensity value is less than 50, a weight 1 is multiplied with the intensity value Else If the intensity value ranges from $51-100$, a weight 2 is multiplied with the intensity value Else If the intensity value ranges from 101-150, a weight .3 is multiplied with the intensity value then calculates the median values. This Enhancement stage, the Weighted Median Filtering is applied for each pixel of $3 \times 3$ window and adjacency pixels are evoked and evaluated the mean gray value of foreground, 


\section{International Journal of Advanced Research in Computer and Communication Engineering}

Vol. 9, Issue 7, July 2020

mean gray value of background and contrast value. If all the weights are equal, then the Weighted Median is the same as the Median Filter. The tumor pixels in the foreground region are white in color their Intensity is 0 to 255 and the background region is normally black in color their intensity is 0 to 10 .

The Contrast of the region is defined by $\mathrm{C}=(\mathrm{f}-\mathrm{b}) /(\mathrm{f}+\mathrm{b})$

Where $\mathrm{f}$ is the mean gray-level value of the foreground $\mathrm{b}$ is the mean gray-level value of the background

\section{Adaptive Filter}

An adaptive filter is a modern filter that Self-adjusts its transfer function according to an optimizing algorithm. A new type of adaptive filter is developed for impulsive noise reduction of an Image without the degradation of an original Image. The design of the filter is adapted to pursue the high contrasted edges of the image.

The Contrast of the region is defined by $\mathrm{C}=(\mathrm{f}-\mathrm{b}) /(\mathrm{f}+\mathrm{b})$

Where $\mathrm{f}$ is the mean gray-level value of the foreground $\mathrm{b}$ is the mean gray-level value of the background

\section{Spatial Filter}
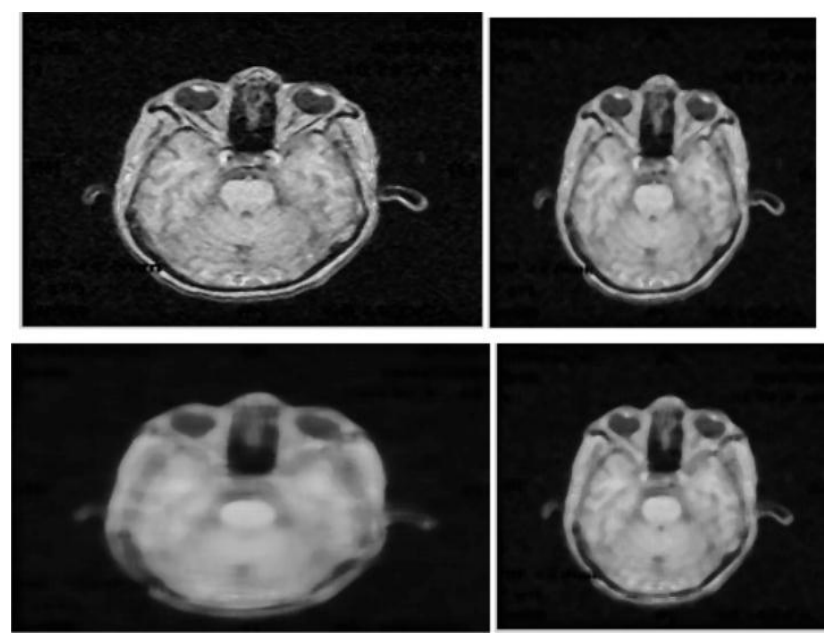

Figure 1.7 Median, Weighted Median, Adaptive and Spatial Filter for MRI
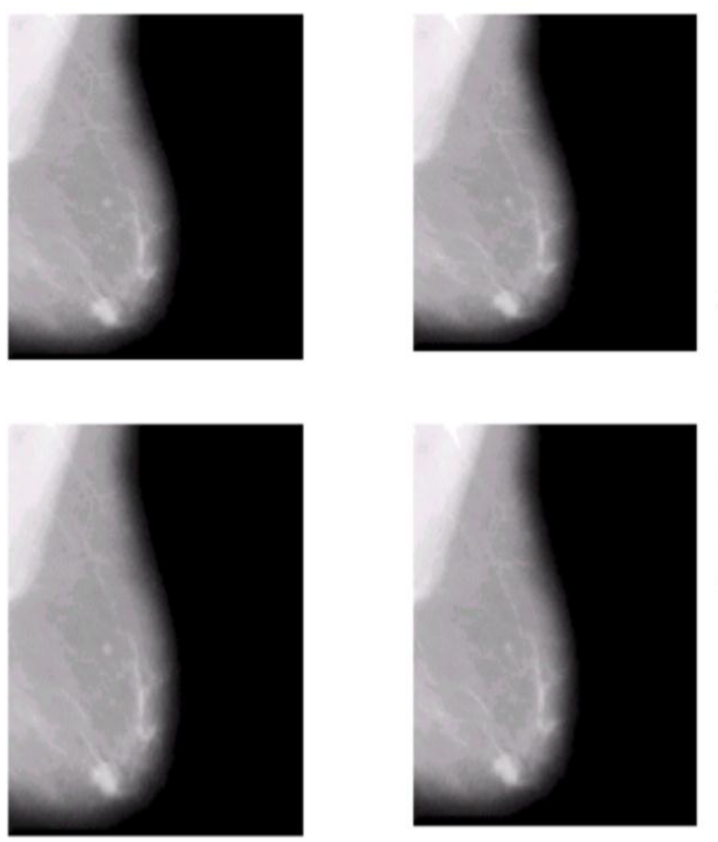

Figure 1.8 Median, Weighted Median, Adaptive and Spatial Filter for Mammogram 
A spatial filter is used to decrease the magnetic noise in the magnetic resonance. This filter is used to abstract the external magnetic noise appearing on MRI scan image and to enhance the signal-to-noise ratio of the MRI brain image. In spatial domain filtering, the filter is notified as 3D array. The kernel applied to the image via convolution or correlation using filter. Here, the filter for the image elements includes a first filter that applies one filter function to the pixels in each column of the image. The partially filtered pixels are stored in matrix and then read row by row in a field interlaced order. The rows of pixel intensity values are sent to a second filter that applies another filter process to each row. The complete filtered image elements from the second filter are stored or transfer to a matrix to display an image. For each pixel, an $3 \times 3$ window of adjacency pixels are extracted A new type of spatial filter is developed for impulsive noise reduction of an image without the degradation of an original image. The shape of the filter basis is adapted to follow the high contrasted edges of the image. In this way, the artifacts introduced by a circularly symmetric filter at the border of high contrasted areas are reduced. And the median value is calculated for that window. Finally $3 \times 3$ window is selected for noise reduction based on high contrast.

\section{EXPERIMENTS AND RESULTS}

It is hard to measure the improvement of the enhancement objectively. If the enhanced image can make the observer perceive the region of interest better, then we can say that the original image has been improved. In order to compare different enhancement algorithms, it is better to design some methods for the evaluation of enhancement objectively. The statistical measurements such as variance or entropy can always measure the local contrast enhancement. However, that shows no consistency for the mammograms.

The Peak Signal-to-Noise Ratio (PSNR) and the Average Signal-to-Noise Ratio (ASNR) are used to evaluate the enhancement performance ( $\mathrm{Li}$ et al. 1996; Li et al. 1997]. The noise level is measured by the standard derivation of the original mammogram image:

$=\operatorname{sqrt}((1 / \mathrm{N}) \mathrm{i}(\mathrm{bi}-\mathrm{b}) 2), \mathrm{i}=1, \ldots, \mathrm{N}$

where $\mathrm{b}$ is the mean gray level value of the original image and bi is the gray level value of a surrounding region, and $\mathrm{N}$ is the total number of pixels in the surrounding region. The PSNR and the ASNR are defined as follows:

$\operatorname{PSNR}=(\mathrm{p}-\mathrm{b}) /$

$\operatorname{ASNR}=(f-b) /$

where $\mathrm{p}$ is the maximum gray level value and $\mathrm{f}$ is the average gray level value of a enhanced image. If the values of the two indices are larger, the proposed preprocessing and enhancement method performance is better. The proposed method produces 0.941 and 0.913 for the PSNR and the ASNR respectively. Table 1.3 compares the performance of the proposed algorithm with the existing methods.

In this analysis, a gradient-based image enhancement method using first derivative and local statistics has been proposed. The final enhanced mammogram image is validated using SNR values. The merit of the gradient-based image enhancement is that it is very simple because enhancing the mammogram image only by local statistics. So the proposed method produces the output faster than any other existing method. Also it produces the same output every time.

\begin{tabular}{|l|l|l|l|}
\hline Authors & Methods & PSNR & ASNR \\
\hline N.R. Mudigonda et al., (2001) & Gaussian smoothening & 0.820 & 0.810 \\
\hline K.J.McLoughlin et al., (2004) & Noise equalization & 0.880 & 0.880 \\
\hline L. Bocchi et al., (2004) & Matched filtering & 0.850 & 0.810 \\
\hline H.D. Cheng et al., (2004) & LOG filtering & 0.870 & 0.860 \\
\hline Thangavel and Karnan (2005) & Pre-processing and Enhancement & 0.910 & 0.930 \\
\hline Logeswari and Karnan (2010) & Pre-processing and Enhancement & 0.911 & 0.944 \\
\hline Logeswari and Karnan (2018) & Pre-processing and Enhancement & 0.912 & 0.943 \\
\hline Proposed Approach & 0.913 & 0.941 \\
\hline
\end{tabular}

\section{CONCLUSION}

Initially the film artifacts are removed and the breast and brain region alone is copied into a new image. With this new image median filtering is applied to remove the high frequency components. The enhanced mammogram image is validated using SNR values. There are four types of filters such as Median Filter, Weighted Median Filter, Adaptive 
Filter and Spatial filter are used to remove high frequency components from MRI Brain Image. The Computational result is used to enhance the Image and the performance of the system was investigated. The result shows that the Weighted Median Filter is better than other filtering techniques. The merit of using Weighted Median Filter, it can remove the noise without disturbing the edges.

\section{REFERENCES}

[1]. Thangavel, K \& Karnan, M 2005, “CAD System for Preprocessing and Enhancement of Digital Mammograms”, GVIP Journal, vol.5, no.9, pp.6974, 2005.J. Breckling, Ed., The Analysis of Directional Time Series: Applications to Wind Speed and Direction, ser. Lecture Notes in Statistics. Berlin, Germany: Springer, 1989, vol. 61.

[2]. Marcel Prastawa, Elizabeth Bullitt \& Guido Gerig, "Simulation of Brain Tumors in MR Images for Evaluation of Segmentation Efficacy",

Medical Image Analysis (MedIA), vol .13, no. 2, pp. 297- 311, 2009.

[3]. Karnan, M \& Logeswari, T, “An Improved Implementation of Brain Tumor Detection using Segmentation Based on Hierarchical Self Organizing Map”, International Journal of Computer Theory and Engineering,vol.2, no.4, pp.591-595, 2010.

[4]. Tripathy, Swarnkar (2018) “A Comparative Analysis on Filtering Techniques used in Pre-processing of Mammogram Image". In 3rd International Conference on Advanced Computing and Intelligent Engineering (ICACIE 2018). Springer.

[5]. Ramani, Vanitha, and Valarmathy. (2013) "The pre-processing techniques for breast cancer detection in mammography images." International Journal of Image, Graphics and Signal Processing 5(5): 47.

[6]. Moradmand, Setayeshi, Karimian, Sirous, and Akbari. (2012) “ Comparing the performance of image enhancement methods to detect microcalcification clusters in digital mammography." Iranian journal of cancer prevention. 5(2):61.

[7]. Sushreeta Tripathy, Tripti Swankar "Unified Preprocessing and Enhancement Technique for Mammogram Images" International Conference on Computational Intelligence and Data Science (ICCIDS 2019) Science Direct

[8]. S.Perumal, T.Velmurugan "Preprocessing by Contrast Enhancement Techniques for Medical Images" International Journal of Pure and Applied Mathematics Volume 118 No. 18 2018, 3681-3688 